\title{
Approximate Analytic Solution of the Heat Equation in the Cast-Mould Heterogeneous Domain by Differential Transformation Method
}

\author{
Mehmet Merdan* and Ahmet Gökdoğan
}

Gümüşhane University, Department of Mathematics Engineering, 29100-Gümüşhane, Turkey

\begin{abstract}
In this article, a new application of Differential Transformation Method (DTM) is presented to find exact and approximate solutions of the heat equation in the cast-mould heterogeneous domain. It is indicated that the solutions obtained by the two dimensional (DTM) are reliable, useful and effective method for decouple partial differential equations. Exact solutions can also be obtained from the known forms of the series solutions.
\end{abstract}

Keywords: Differential transformation method; Approximate analytic solutions; The heat equation in the cast-mould heterogeneous domain

\section{Introduction}

In the current paper, we consider the problem of determining distribution of temperature in the heat equation in the cast-mould heterogeneous domain. Let's start by formulating the first mathematical model of the problem. $D_{1}$ and $D_{2}$ regions are given below (Figure 1):

$$
\begin{aligned}
& D_{1}=\left\{(x, t): x \in\left[x_{1}, 0\right], t \in\left[0, t^{*}\right)\right\} \\
& D_{2}=\left\{(x, t): x \in\left[0, x_{2}\right], t \in\left[0, t^{*}\right)\right\}
\end{aligned} \text { and }
$$

Five-component boundaries of this region are dispatched:

$$
\begin{aligned}
& \Gamma_{1}=\left\{(x, 0): x \in\left[x_{1}, 0\right]\right\}, \\
& \Gamma_{2}=\left\{(x, 0): x \in\left[0, x_{2}\right]\right\}, \\
& \Gamma_{3}=\left\{\left(x_{1}, t\right): x \in\left[0, t^{*}\right)\right\}, \\
& \Gamma_{4}=\left\{(0, t): t \in\left[0, t^{*}\right)\right\}, \\
& \Gamma_{5}=\left\{\left(x_{2}, t\right): t \in\left[0, t^{*}\right)\right\},
\end{aligned}
$$

In the cast (domain $D_{1}$ ) and mould (domain $D_{2}$ ) we consider the heat transfer equations:

$$
\begin{aligned}
& \frac{\partial u(x, t)}{\partial t}=a_{1} \frac{\partial^{2} u(x, t)}{\partial x^{2}} \text { in } D_{1} \\
& \frac{\partial v(x, t)}{\partial t}=a_{2} \frac{\partial^{2} v(x, t)}{\partial x^{2}} \text { in } D_{2}
\end{aligned}
$$

Where $a_{i}, i=1,2$, are the thermal diffusivity, $u, v$ denote the temperature, and $t$ and $x$ indicate the time and spatial location. At the boundaries of the above functions satisfy the initial and boundary conditions:

$$
\begin{aligned}
& u(x, 0)=\varphi_{1}(x), \quad x \in \Gamma_{1}, \\
& v(x, 0)=\varphi_{2}(x), \quad x \in \Gamma_{2}, \\
& u\left(x_{1}, t\right)=\psi(t), \quad t \in \Gamma_{3}, \\
& v\left(x_{2}, t\right)=q(t), \quad t \in \Gamma_{5}, \\
& u(0, t)=v(0, t), \quad t \in \Gamma_{4}, \\
& -\lambda_{1} \frac{\partial u(0, t)}{\partial x}=-\lambda_{2} \frac{\partial v(0, t)}{\partial x}, \quad t \in \Gamma_{4},
\end{aligned}
$$

where the $\lambda_{i}, i=1,2$, denote the thermal conductivity.
The investigation of the exact and numerical solution to nonlinear equations plays an important role in the study of nonlinear physical phenomena. The modified KdV types of equations have been an important class of non-linear evolution equations with numerous applications in physical sciences and engineering fields. For example, in geophysical fluid dynamics, they describe a long wave in shallow seas and deep oceans [1-3]. In plasma physics these equations give rise to the ion acoustic solutions [4-6]. However, the physical situations in which the $\mathrm{KdV}$ equations arise tend to be highly idealized due to the assumption of constant coefficients. In the recent years, many authors mainly had paid attention to study solutions of coupled equations by using various methods. Among these are Trigonometric function transform method [7], the homogeneous balance method [8], the F-expansion transform method [9], the He's variational iteration method [10-13], Homotopy perturbation method [14,15], Adomian decomposition method $[16,17]$ and other [18-20].

In this paper, the differential transformation method (DTM) [2128 ] is used to solve the coupled-mKdV equations.

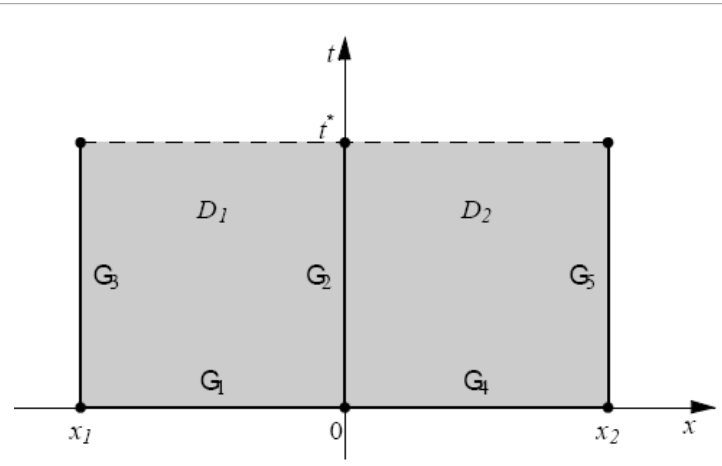

Figure 1: Domain of discussed problem

*Corresponding author: Mehmet Merdan, Gümüşhane University, Department of Mathematics Engineering, 29100-Gümüşhane, Turkey, E-mail: mmerdan@gumushane.edu.tr

Received March 03, 2012; Accepted May 22, 2012; Published May 25, 2012

Citation: Merdan M, Gökdoğan A (2012) Approximate Analytic Solution of the Heat Equation in the Cast-Mould Heterogeneous Domain by Differential Transformation Method. J Appl Computat Math 1:111. doi:10.4172/2168-9679.1000111

Copyright: ( $) 2012$ Merdan M. This is an open-access article distributed under the terms of the Creative Commons Attribution License, which permits unrestricted use, distribution, and reproduction in any medium, provided the original author and source are credited. 
This paper is organized as follows:

In section 2, we describe DTM briefly. To show in efficiency of this method, we give the implementation of the DTM for the coupled$\mathrm{mKdV}$ equation and numerical results in Section 3. The conclusions are then given in the final section 4 .

\section{Differential Transformation Method}

The basic definitions and fundamental operations of the two dimensional differential transform function of the function are expressed as follows $[21,22,25]$. Two dimensional differential transform of $u(x, y)$ is the following form

$$
U(k, h)=\frac{1}{k ! h !}\left(\frac{\partial^{k+h} u(x, y)}{\partial x^{k} \partial y^{h}}\right)_{\left(x_{0}, y_{0}\right)}
$$

where $u(x, y)$ is the original function and $U(k, h)$ is the transformed function. The inverse differential transform of $U(k, h)$ is defined as

$$
u(x, y)=\sum_{k=0}^{\infty} \sum_{h=0}^{\infty} U(k, h)\left(x-x_{0}\right)^{k}\left(y-y_{0}\right)^{k}
$$

when $\left(x_{0}, y_{0}\right)$ are taken as $(0,0)$, the function $u(x, y)$, Eq.(7), is showed as the following

$$
u(x, y)=\sum_{k=0}^{\infty} \sum_{h=0}^{\infty} \frac{1}{k ! h !}\left[\frac{\partial^{k+h} u(x, y)}{\partial x^{k} \partial y^{h}}\right]_{(0,0)} x^{k} y^{k} .
$$

From the above definitions, it can be found that the concept of twodimensional differential transform is derived from two-dimensional differential transform is obtained from two-dimensional Taylor series expansion.

\section{The DTM Applied to the Heat Equation in the Cast- Mould Heterogeneous Domain}

In this section, we will investigate the solution of the heat equation in the cast-mould heterogeneous domain, which have been widely examined in the literature. We described the implementation of the DTM the heat equation in the cast-mould heterogeneous domain in detail. Application of the presented procedure will be examined with the help of parameters values, in which $x_{1}=-1, x_{2}=1, a_{1}=\frac{1}{4}, a_{2}=1, \lambda_{1}=1$ and $\lambda_{2}=2$ To solve Eqs (3)-(5), according to DTM, Eqs. (5) with initial condition,

$$
u(x, 0)=e^{2 x}, v(x, 0)=e^{x}
$$

the boundary conditions,

$$
u(-1, t)=e^{t-2}, v(1, t)=e^{t+1},
$$

applying the differential transform of (3), (4), (9) and(10), then

$$
(h+1) U(k, h+1)=a_{1}(k+1)(k+2) U(k+2, h)
$$

$(h+1) V(k, h+1)=a_{2}(k+1)(k+2) V(k+2, h)$

$$
\begin{aligned}
& U(k, 0)=\frac{2^{k}}{k !}, V(k, 0)=\frac{1}{k !} \\
& U(-1, k)=\frac{e^{-2}}{k !}, V(1, k)=\frac{e}{k !},
\end{aligned}
$$

Substituting Eq. (11)-(12) into Eq. (13)-(14), we obtain the closed form solution as

$$
\begin{aligned}
& u(x, t)=\sum_{k=0}^{\infty} \sum_{h=0}^{\infty} U(k, h) x^{k} t^{h}=1+t+\frac{t^{2}}{2}+2 x+2 x t+x t^{2} \\
&+ 2 x^{2}+2 x^{2} t+x^{2} t^{2}+\frac{4}{3} x^{3}+\frac{4}{3} x^{3} t+\frac{2}{3} x^{3} t^{2}+\ldots=e^{t+2 x} \\
& v(x, t)=\sum_{k=0}^{\infty} \sum_{h=0}^{\infty} V(k, h) x^{k} t^{h}=1+t+\frac{1}{2} t^{2}+x+x t+\frac{1}{2} x t^{2}+\frac{1}{2} x^{2} \\
&+\frac{1}{2} x^{2} t+\frac{1}{4} x^{2} t^{2}+\frac{1}{6} x^{3}+\frac{1}{6} x^{3} t+\frac{1}{12} x^{3} t^{2}+\ldots=e^{t+x}
\end{aligned}
$$

which are the exact solutions [6]. The graph of exact and DTM solutions belonging to examples examined the above are shown at Figures 1-2 for $\lambda_{1}=1$ and $\lambda_{2}=2$. it can be deduced that DTM solution corresponds to the exact solutions (Figure 2 and 3 ).

\section{Conclusions}

In this article, to find the numerical solution of the heat equation in the cast-mould heterogeneous domain with a number of initial and boundary values, the two dimensional differential transformation method (DTM) was has been performed successfully application. The results obtained from DTM show that in full compliance with exact solution. The solution obtained by differential transformation method shown as an expression of the form of a series of the exact solution.

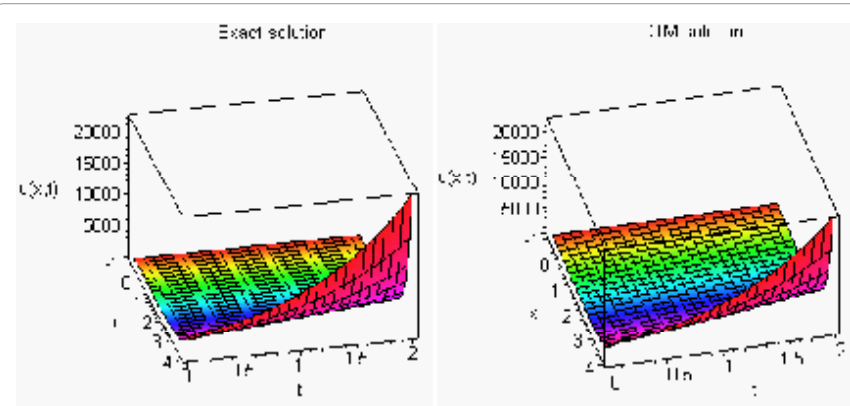

Figure 2: Exact solution and DTM solutions for $\mathrm{u}(x, t)$ when for $\lambda_{1}=1$ and $\lambda_{2}=2$.

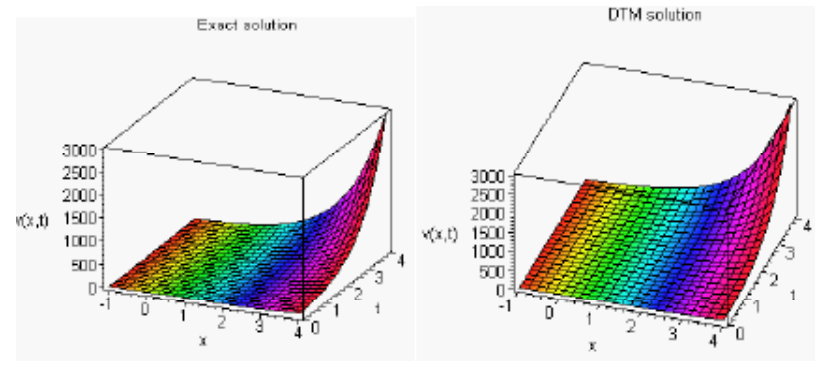

Figure 3: Exact solution and DTM solutions for $u(x, t)$ when for $\lambda_{1}=1$ and $\lambda_{2}=2$ 


\begin{tabular}{|l|l|}
\hline $\begin{array}{l}\text { Original function } \\
u(x, y)=f(x, y) \mp g(x, y)\end{array}$ & Transformed function \\
\hline$u(x, y)=\xi f(x, y)$ & $U(k, h)=\xi F(k, h)$ \\
\hline$u(x, y)=\frac{\partial f(x, y)}{\partial x}$ & $U(k, h)=(k+1) F(k+1, h)$ \\
\hline$u(x, y)=\frac{\partial^{m+n} f(x, y)}{\partial x^{m} \partial y^{n}}$ & $U(k, h)=(k+1) \ldots(k+m)(h+1) \ldots(h+n) F(k+m, h+n)$ \\
\hline$u(x, y)=x^{r} y^{s}$ & $U(k, h)=\delta(k-r, h-s)=\left\{\begin{array}{l}1, \quad k=r, h=s \\
0, \quad \text { otherwise }\end{array}\right.$ \\
\hline$u(x, y)=f(x, y) g(x, y)$ & $U(k, h)=\sum_{m=0}^{k} \sum_{n=0}^{h} F(m, h-n) G(k-m, n)$ \\
$u(x, y)=f(x, y) g(x, y) h(x, y)$ & $U(k, h)=\sum_{k_{4}=0}^{k} \sum_{k_{3}=0}^{k-k_{4}} \sum_{k_{2}=0}^{h} \sum_{k_{1}=0}^{h-k_{2}} F\left(k_{4}, h-k_{2}-k_{1}\right)$ \\
$k\left(k-k_{4}-k_{3}, k_{1}\right)$
\end{tabular}

Table 1: Operations of the two dimensional differential transform.

DTM can be applied to many complicated linear and strongly nonlinear partial differential equations and does not require linearization, discretization or perturbation. The results of the present method show that this method is useful and effective for solving in the cast-mould heterogeneous domain.

\section{References}

1. Wu Y, Geng X, Hu X, Zhu S (1999) A generalized Hirota--Satsuma coupled Korteweg--de Vries equation and Miura transformations. Phys Lett A 255: 259 264.

2. Ostrovsky L, Stepanyants YuA (1989) Do internal solutions exist in the ocean? Rev Geophys 27: 293-310.

3. Cao DB, Yan JR, Zhang Y (2002) Exact solutions for a new coupled MKdV equations and a coupled KdV equations. Phys Lett A 297: 68-74.

4. Das GC, Sarma J (1999) Response to comment on 'A new mathematical approach for finding the solitary waves in dusty plasma'. Phys Plasmas 6: 4394-4397.

5. Gao YT, Tian B (2001) lon-acoustic shocks in space and laboratory dusty plasmas: Two-dimensional and non-traveling-wave observable effects. Phys Plasmas 8: 3146-3149.

6. Hirota R, Satsuma J (1981) Solition solutions of a coupled Korteweg-de Vries equation. Phys Lett A 85: 407-408.

7. Osborne AR (1995) The inverse scattering transform: Tools for the nonlinear fourier analysis and filtering of ocean surface waves. Chaos, Solitons \& Fractals 5: 2623-2637.

8. Zayed EME, Zedan HA, Gepreel KA (2004) On the solitary wave solutions for non-linear Hirota-Satsuma coupled-KdV of equations. Chaos, Solitons \& Fractals 22: 285-303.

9. Zhang JL, Wang ML, Wang YM, Feng ZD (2006) The improved F-expansion method and its applications. Phys Lett A 350: 103-109.

10. Assas LMB (2008) Variational iteration method for solving coupled-KdV equations. Chaos, Solitons and Fractals 38: 1225-1228.
11. Ganji DD, Jannatabadi M, Mohseni E (2007) Application of He's variationa iteration method to nonlinear Jaulent-Miodek equations and comparing it with ADM. J Comput Appl Math 207: 35-45.

12. $\mathrm{He} \mathrm{JH}, \mathrm{Wu} \mathrm{XH}$ (2006) Construction of solitary solution and compacton-like solution by variational iteration method. Chaos, Solitons Fractals 29: 108-113.

13. Guo-Zhong Z, Xi-Jun Y, Yun X, Jiang Z, Di W (2010) Approximate analytic solutions for a generalized Hirota Satsuma coupled $\mathrm{KdV}$ equation and a coupled mKdV equation. Chin Phys B 19: 1-9.

14. Ganji DD, Rafei M (2006) Solitary wave solutions for a generalized HirotaSatsuma coupled-KdV equation by homotopy perturbation method. Phys Lett A 356: 131-137.

15. Yıldırım A (2008) The homotopy perturbation method for approximate solution of the modified KdV equation. Z Naturforsch 63a: 621-626.

16. Raslan KR (2004) The decomposition method for a Hirota-Satsuma coupled $\mathrm{KdV}$ equation and a coupled mKdV equation. Int J Comput Math 81: 1497 1505

17. Bildik N, Konuralp A (2006) The use of variational iteration method, differential transform method and Adomian decomposition method for solving different types of nonlinear partial differential equations. Int J Nonlin Sci Num 7: 65-70.

18. Kaya D, El-Sayed SM (2003) A numerical method for solving Jaulent-Miodek equation. Phys Lett A 318: 3450-3453.

19. Fan E (2001) Soliton solutions for a generalized Hirota-Satsuma coupled KdV equation and a coupled MKdV equation. Phys Lett A 282: 18-22.

20. Abassy TA, El-Tawil MA, Zoheiry HE (2007) Toward a modified variational iteration method. J Comput Appl Math 207: 137-147.

21. Zhou JK (1986) Differential transform and its applications for electrical circuits Huazhong University Press, Wuhan, China.

22. Chen CK, Ho SH (1999) Solving partial differential equations by two-dimensional differential transform method. Appl Math Comp 106: 171-179.

23. Jang MJ, Chen CL, Liu YC (2001) Two-dimensional differential transform for partial differential equations. Appl Math Comp 121: 261-270.

24. Hassan AH (2002) Different applications for the differential transformation in the differential equations. Appl Math Comp 129: 183-201. 
Citation: Merdan M, Gökdoğan A (2012) Approximate Analytic Solution of the Heat Equation in the Cast-Mould Heterogeneous Domain by Differential Transformation Method. J Appl Computat Math 1:111. doi:10.4172/2168-9679.1000111

Page 4 of 4

25. Ayaz F (2003) On two-dimensional differential transform method. Appl Math Comp 143: 361-374.

26. Ayaz F (2004) Solution of the system of differential equations by differential transform method. Appl Math Comp 147: 547-567.
27. Chang SH, Chang IL (2009) A new algorithm for calculating two-dimensional differential transform of nonlinear functions. Appl Math Comp 215: 2486-2494.

28. Kurnaz A, Oturnaz G, Kiris ME (2005) n-Dimensional differential transformation method for solving linear and nonlinear PDE's. Int J Comp Math 82: 369-380. 